\title{
Latest results from the PolarquEEEst missions
}

\section{Marcello Abbrescia ${ }^{a, b, *}$ on behalf of the EEE Collaboration}

(a complete list of authors can be found at the end of the proceedings)

${ }^{a}$ Dipartimento Interateneo di Fisica, Università di Bari, Bari, Italy

${ }^{b}$ INFN Sezione di Bari, Bari, Italy

E-mail: marcello.abbrescia@ba.infn.it

The PolarquEEEst scientific programme consists in a series of measurements of the cosmic ray flux up to the highest latitudes, well beyond the Polar Article Circle. It started in Summer 2018, when one telescope for cosmic rays was installed on a sailboat leaving from North Iceland, to circumnavigate the Svalbard archipelago and land in Troms $\varnothing$. It collected data up to $82 \mathrm{~N}$, measuring with unprecedented precision the charged particle rate at sea level in these regions.

During Fall of the same year and Spring 2019 the PolarquEEEst programme continued with a series of measurements performed using the same detector, which took place first in Italy, with the southernmost point reached at Lampedusa, and then in Germany, with the goal to measure the dependence of cosmic charged particle rate with latitude.

Then, in May 2019, the PolarquEEEst collaboration accomplished another important result, installing a cosmic ray observatory for the detection of secondary cosmic muons at Ny Alesund, at $79 \mathrm{~N}$, made of three independent identical detectors positioned a few hundred meters from each other, and synchronized in order to operate together as a network. This configuration allows high precision measurements never performed before at these latitudes on a long term, also interesting for their connection with environmental phenomena.

Here the various missions will be presented, and the latest results from the measurements performed will be shown.

$37^{\text {th }}$ International Cosmic Ray Conference (ICRC 2021)

July 12th - 23rd, 2021

Online - Berlin, Germany

\footnotetext{
*Presenter
} 


\section{Introduction}

The PolarquEEEst experiment was designed to perform measurements on the flux of cosmic rays. It started its data acquisition with the PolarquEEEst2018 campaign, when the cosmic charged particle rate at sea level in a latitude range between $66 \mathrm{~N}$ and $82 \mathrm{~N}$ was measured with unprecedented precision. In that occasion, a detector was hosted on the 60- feet eco-friendly sailboat Nanuq, which left for a six weeks cruise on July 22, 2018, from Ísafjörður (Iceland), reached Longyearbyen (Svalbard Archipelago, Norway), then circumnavigated the Svalbard Archipelago reaching the maximum latitude of $8207 \mathrm{~N}$, well beyond the Polar Arctic Circle, and ended its voyage on September 4, in Troms $\varnothing$ (Norway) [1] [2]. In addition to the detector on board the sailboat Nanuq, two other identical detectors were located in Norway and in Italy and used for cross-reference.

The PolarquEEEst missions continued with a series of measurements in Italy, southward reaching Lampedusa, and in Germany, to measure the flux of cosmic rays on the ground as a function of latitude. Finally, in May 2019, the PolarquEEEst collaboration installed a cosmic ray observatory for the detection of secondary cosmic muons at $\mathrm{Ny}$ Alesund, at $79^{\circ} \mathrm{N}$, made of three independent detectors positioned a few hundred meters from each other, and synchronized in order to operate together as a network [3]. The configuration used allows high precision measurements never performed before at these latitudes on a long term, also interesting for their connection with environmental phenomena. The network also complements the existing stations for the detection of cosmic neutrons at the Svalbard archipelago, enlarging the physics scope that is possible to pursue in this field at this peculiar location.

Here the detector layout and characteristics are described. Then the various missions where the POLA detectors were used are discussed, and some results are presented.

\section{The PolarquEEEst detectors}

The detectors for PolarquEEEst, conventionally called POLA detectors, were developed respecting the constraints in terms of dimensions, weight, power consumption, and the requirements on robustness and reliability deriving from the fact that one of them had to be hosted on board of a sailing boat, cruising for six weeks with limited support and possibility to replace components. In particular, due to the eco-friendly nature of the mission, the total electrical power available on the boat was limited, and the one assigned to the PolarquEEEst detector on board was less than $20 \mathrm{~W}$. Nevertheless the detectors had to be characterized by high efficiency and by the possibility to precisely tag the events in time and space in order to produce a detailed map of the cosmic ray intensity during the trip.

Sensitive elements of the PolarquEEEst detectors are $1 \mathrm{~cm}$ thick planes of BC400 Saint-Gobain plastic scintillators, each made out of four tiles $20 \times 30 \mathrm{~cm}^{2}$ in dimensions, as shown in Figure 1 . Each tile is read out by means of two AdvanSid ASD-NUV4S-P-40 Silicon PhotoMultipliers (SiPMs). Each POLA detector comprises two scintillator planes, separated by $11 \mathrm{~cm}$, enclosed in a light-tight box.

The SiPMs are readout by Front End electronics (FE) custom cards, each able to independently process the analog signals from two SiPMs. Each card includes two input pre-amplifiers, and 


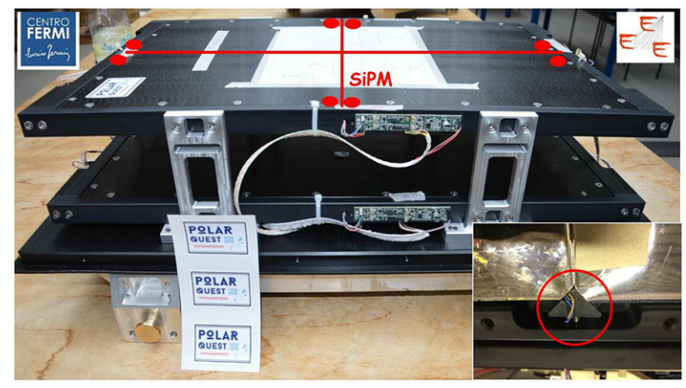

Figure 1: One of the three PolarquEEEst detectors. The lines indicate the 4 scintillator tiles and the red points the position of the SiPMs. The small insert shows the cut on the scintillators and the wedges carved into the scintillators to ensure a good contact to the SiPM.

discriminators, whose thresholds can be remotely adjusted. Output from the FE card are lowvoltage differential signals, fed to a custom Trigger and Read-out Board (TRB) [5].

The TRB is equipped with an Altera Cyclone 5 Field Programmable Gate Array (FPGA), a high performance Multi-Hit Time-to-Digital Converter (HPTDC), and an USB card with a FT232H chip. The trigger logic requires the coincidence in a $10 \mathrm{~ns}$ window of two signals coming from two SiPMs reading the same scintillator tile and an additional signal coming from a SiPM from a scintillator of the other plane, and is implemented in the FPGA. The electronics also includes a GPS (Global Positioning System) and a GLONASS (GLObal NAvigation Satellite System) boards used to provide the position of the device and a timestamp to each event recorded.

A complete description of POLA detectors can be found in [4].

\section{Detector calibration and tests}

After assembling, the POLA detectors were tested for a month long period, at CERN. Usual basic checks were performed by verifying that the measured frequencies of the impinging particles were roughly consistent across all the detectors. At this stage, also, the thresholds and bias voltages of all SiPM channels were precisely set by slightly tuning them with respect to their default values, in order to get the Time-Over-Threshold (TOT) distributions similar for all channels in all detectors.

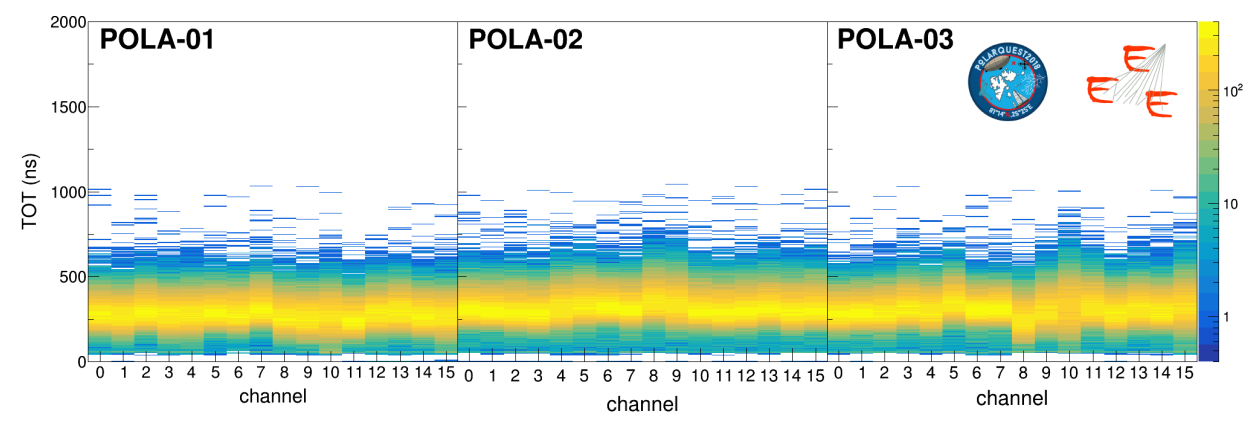

Figure 2: TOT distributions for POLA-01, POLA-02 and POLA-03, for data taken on July 27, 2018. Here each bin corresponds to one SiPM. 


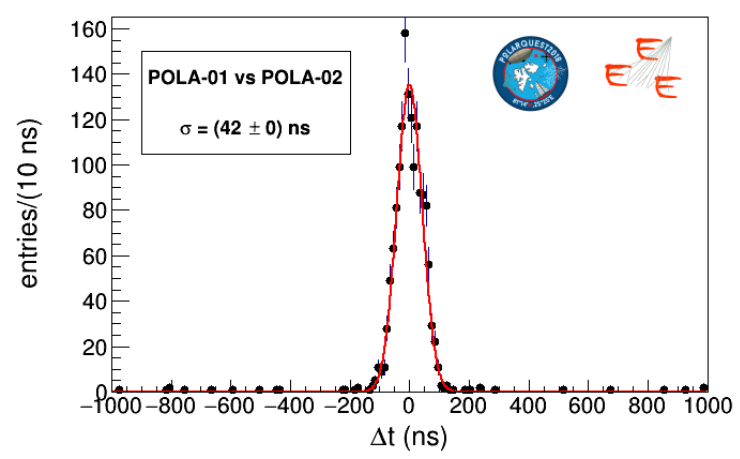

Figure 3: Time coincidences between POLA-01 and CERN-01, for data taken on the June 17, 2018.

Examples of TOT distributions after equalization are shown in Fig. 2; they are quite similar across the various channels of the POLA detectors. Here each bin refers to a SiPM of each detector.

During the period when the detectors stayed at CERN, the GPS time tags, in particular, were carefully checked, in order to be sure that the time reconstruction chain worked properly. A plot of the events detected in coincidence by POLA-01 and POLA-02 is reported in Fig. 3. The plot refers to events acquired on June 17, 2018, for a period of about 11 hours. In the figure the peak due to the particles associated to the same Extensive Air Shower impinging on both detectors can be clearly seen above the background due to accidentals. The observed width of the peak, around $40 \mathrm{~ns}$, is due to two main contributions: the intrinsic time precision of the two detectors, and the time spread due to the uncertainty on the arrival direction of the shower front.

\section{Results from the PolarquEEEst2018 mission}

The raw rate measured by POLA-01 during its trip is shown in Figure 4, together with the detector geographic and magnetic latitudes, and geographic longitude, as a function of time. POLA01 reached the maximum latitude, slightly above $82^{\circ} \mathrm{N}$, in the evening of August 13, 2018. From

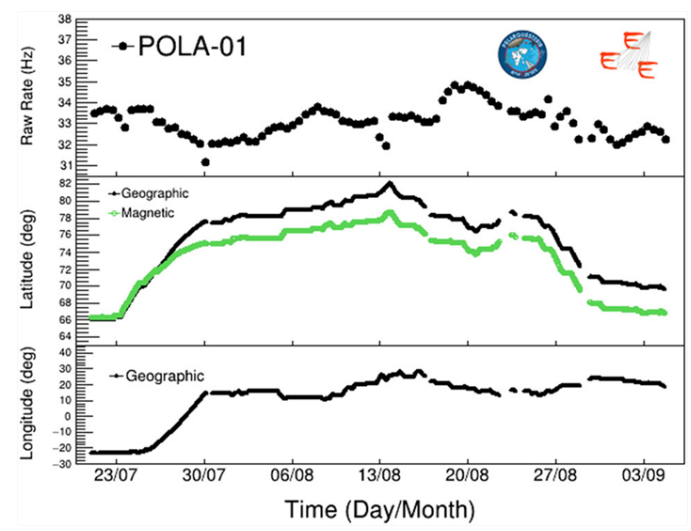

Figure 4: POLA-01 raw rate (top panel), geographic and magnetic latitude (middle panel) and longitude (bottom panel) as a function of the time since July 22, 2018, till the end of its trip in Troms $\varnothing$. 


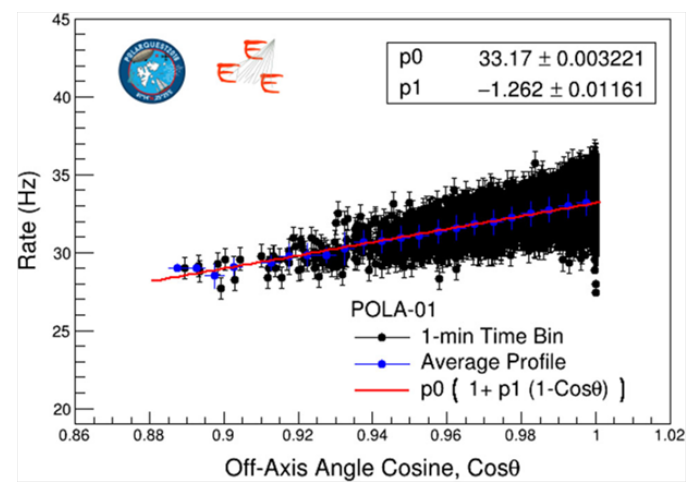

Figure 5: POLA-01 rate as a function of the cosine of the angle between the vertical and the axis perpendicular to the top surface of the detector.

this Figure there is no evidence of any correlation of the measured raw rate with geographical or magnetic coordinates.

However, in order to draw reliable conclusions about a possible dependence of the observed rate of cosmic particle on the latitude, all local effects which can affect such measurement have to be taken into consideration. One important factor to be taken into account for POLA-01 is its variable inclination with respect to the vertical direction, due to the fact that it was mounted on a sailing boat which stayed inclined for long periods of time because of the sea conditions or the sailing pace. Detector inclination affects the measured raw rate because of its angular acceptance and the fact that the cosmic flux at sea level depends on the zenithal angle, namely a larger number of cosmic particles comes from directions close to the vertical.

It has been verified that, in the angular range of interest here, the measured rate follows a simple $\cos \theta$ law, as shown in Fig. 5. Therefore, the raw rate was corrected by dividing the raw rate for the cosine of the inclination angle.

Also a correction to the measured raw rates, associated to the instantaneous atmospheric pressure, has to be applied. An increase in pressure, in fact, causes a reduction of the observed rate because cosmic secondary particles have to traverse more material from their production high up in the atmosphere before reaching the ground. POLA-01 rate measured during the whole data taking period before and after pressure correction is shown in Fig. 6.
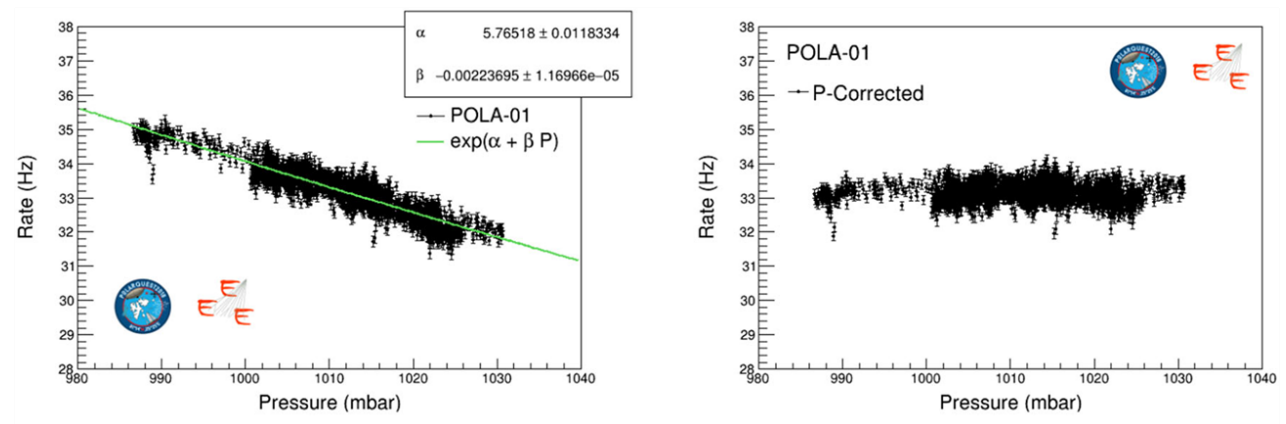

Figure 6: (Left): POLA-01 rate as a function of pressure; (Right): same, but after the barometric correction. 


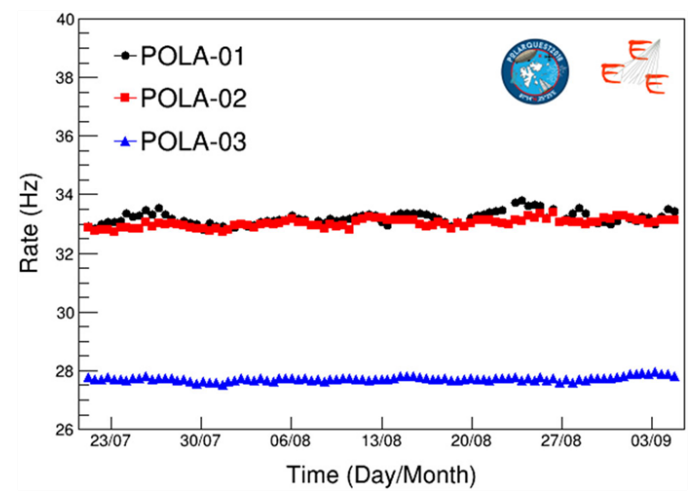

Figure 7: Rates of cosmic particles measured by the three POLA detectors, corrected for the orientation (POLA-01) and pressure (POLA-01, POLA-02 and POLA-03) effects, as a function time.

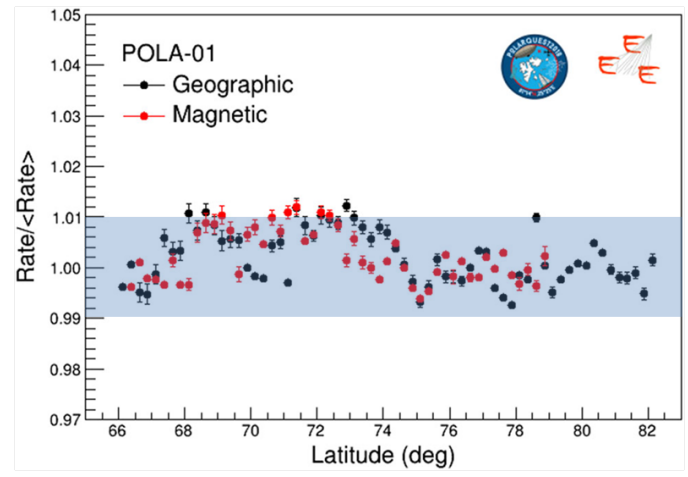

Figure 8: Rate measured by POLA-01, divided by the average rate, as a function of geographic (black points) and geomagnetic (red points) latitude, once all corrections have been applied. All data points fall within a $\pm 1 \%$ band around the average.

The rates of cosmic particles measured by POLA-01, POLA-02 and POLA-03 during the PolarqueEEEst mission, corrected for the pressure and inclination effects just cited, are shown in Fig. 7 as a function of time. No other correction, for instance due either to the thickness of the materials above the detectors, or their different altitudes, or their efficiency, was applied. This explains why POLA-03, which was positioned under a thick roof of bricks, measured a lower rate than the other two detectors. Rates stay around an average value of $33.18 \mathrm{~Hz}$ for POLA-01, 33.03 $\mathrm{Hz}$ for POLA-02 and $27.73 \mathrm{~Hz}$ for POLA-03, with small fluctuations with respect to these values.

Figure 8 summarizes the results of the PolarquEEEst2018, showing the cosmic particle rate, divided by the average rate, measured by POLA-01 on board of the sailboat Nanuq, as a function of latitude, once all corrections have been applied. Both geographic and geomagnetic latitudes are considered here. Indeed, in the very northern regions, geographical and geomagnetic latitudes can significantly differ, and this is confirmed, for instance, by the fact that the northernmost geomagnetic latitude reached by the Nanuq was less than $80^{\circ} \mathrm{N}$, to be compared with the $82^{\circ} \mathrm{N}$ of geographic latitude. No clear evidence of any trend of the rate vs. geographic or geomagnetic latitude is visible in Figure 8, and a uniform behaviour is found within an unprecedented precision of $\pm 1 \%$. 
Table 1: The various stops of the POLA-01 detector during its trip in Italy and Germany at the end of 2018 and first months of 2019.

\begin{tabular}{lll}
\hline Location & Date & Latitude North \\
\hline Genova & October 25, 2018 & $44^{\circ} 24^{\prime}$ \\
Vigna di Valle (Rome) & November 27, 2018 & $42^{\circ} 04^{\prime}$ \\
Cosenza & December 3, 2018 & $39^{\circ} 18^{\prime}$ \\
Messina & December 5, 2018 & $38^{\circ} 11^{\prime}$ \\
Cefalú (Palermo) & December 6, 2018 & $38^{\circ} 02^{\prime}$, \\
Erice $(\approx$ 900 m a.s.l., Trapani) & December 6-8, 2018 & $38^{\circ} 02^{\prime}$, \\
Catania-Etna $\approx$ 2000 m a.s.l.) & December 12, 2018 - February 15, 2019 & $37^{\circ} 30^{\prime}$ \\
Lampedusa & March 6-15, 2019 & $35^{\circ} 30^{\prime}$ \\
Bologna & April 3-4, 2019 & $44^{\circ} 29^{\prime}$ \\
Munich & April 10, 2019 & $48^{\circ} 08^{\prime}$ \\
Hannover & April 10-11, 2019 & $52^{\circ} 22^{\prime}$ \\
Frankfurt am Main & April 11-12, 2019 & $50^{\circ} 06^{\prime}$ \\
CERN & April 12 - May 2, 2019 & $46^{\circ} 12^{\prime}$ \\
\hline
\end{tabular}

\section{The PolarquEEEst journey in Italy and Germany}

In Fall 2018, after the dismounting of the POLA-01 detector from its location on-board the sailboat Nanuq, a new campaign of measurements with the POLA detectors was started. Goal of this campaign was to explore a range of latitudes where the cosmic ray flux at sea level is expected to have an important dependence on latitude and to evaluate the amount of such a dependence. For this purpose, the POLA-01 detector was mounted onto a car, and transported across Italy and Germany, taking data on programmed stops during the trip. The detector did not receive any modification with respect to the configuration adopted for the previous data taking campaign, except that its power system was modified in order to be powered up with a standard 220V AC current.

POLA-01 collected data on multiple occasions, in a variety of conditions. Usually the periods of data taking ranged from several hours to a couple of days. Locations, periods of data taking and the corresponding geographic latitudes are listed in Table 1. The first part of this data taking campaign was in Fall 2018, when POLA-01 left from Genova, southward to arrive at Lampedusa, the southernmost location in Italy, and then back to Bologna. Then, in order to cover more of the region of interest, the second part was done in April 2019, transporting the POLA-01 detector by car and leaving from Bologna, to Munich, Hannover, Frankfurt am Main, to finish the trip at CERN, where the detector collected data for another twenty days.

Analysis on the data taken during this trip is underway.

\section{The PolarquEEEst2019 mission}

In May 2019 three POLA detectors were installed at the Ny-Ålesund international Research Station, located at the Svalbard Archipelago, in order to start a long term study of the high-energy cosmic ray flux with charged particles at sea level and at the northernmost latitudes. 
For these studies, a configuration envisaging a mini-array of three POLA detectors, located at few hundred meters from each other, synchronized via the GPS signals and operating together as a network, was chosen. It this way the POLA detectors can provide a time stamp to each recorded event at a few nanosecond precision. This allows to search for correlations in time among the events recorded at the three stations, making it possible to identify very high energy Extensive Atmospheric Showers (EAS) with a front at the ground large enough to impinge at the same time in the whole region of interest. Also the low energy background component, unable to provide events in coincidence on two or three stations, would be rejected. Moreover, using a network of three detectors, makes it possible, by means of triangulation, to reconstruct the direction of arrival of the cosmic radiation, opening the possibility to search for any anisotropy in the cosmic radiation distribution, particularly interesting so close to the magnetic North Pole.

In addition to operating as a network, the three detectors can perform interesting measurements locally, i.e. at the level of the single detector, measuring just the muon flux and its variations in connection with transient astrophysical phenomena, among them the detection of Forbush decreases connected with Solar Flare followed by Coronal Mass Emissions, or providing alerts for Ground Level Enhancement of Solar cosmic rays.

The position of the three detectors was dictated by the available infrastructure, in particular the presence of buildings with heating, electric power and connection to the internet. At Ny-Ålesund three sites satisfying the requirements above, and available to host the three POLA detectors, were identified: a small wooden hut close to the Amundsen-Nobile Climate Change Tower where POLA01 was decided to be located, the "Dirigibile Italia" station, managed by the Consiglio Nazionale delle Ricerche (CNR), that was to host POLA-03, and the Gruvebadet Laboratory for POLA-04.

Since May 2019 the POLA detectors are regularly taking data, and the analysis, in particular looking for correlation with interesting atmospheric and astrophysical phenomena in ongoing, possibly opening new interesting perspectives in this research field.

\section{References}

[1] http://wwW . polarquest2018.org

[2] R. Nania and O. Pinazza, Measuring cosmic ray showers near the North Pole with the Extreme Energy EventsProject, Nuovo Sagg. 34 (2018) 27.

[3] M. Abbrescia et al. (EEE collaboration), Results from the PolarquEEEst missions, J. Phys.: Conf. Ser. 1561 012001, 2020, doi:10.1088/1742-6596/1561/1/012001

[4] M. Abbrescia et al. (EEE collaboration), New high precision measurements of the cosmic charged particle rate beyond the Arctic Circle with the PolarquEEEst experiment. Eur. Phys. J. C (2020) 80: 665 https://doi.org/10.1140/epjc/s10052-020-8213-2

[5] R. Travaglini et al., A multi-channel trigger and acquisition board for TDC-based readout: application to the cosmic rays detector of the PolarQuEEEst 2018 project, PoS(TWEPP2019)150 


\section{Full Authors List: EEE Collaboration}

M. Abbrescia ${ }^{1,2}$, C. Avanzini ${ }^{3}$, L. Baldini ${ }^{3,4}$, R. Baldini Ferroli ${ }^{5}$, G. Batignani ${ }^{3,4}$, M. Battaglieri ${ }^{6,7}$, S. Boi ${ }^{8,9}$, E. Bossini ${ }^{3,4}$, F. Carnesecchi ${ }^{10,11}$, C. Cicalò ${ }^{9}$, L. Cifarelli ${ }^{10,11}$, F. Coccetti ${ }^{12}$, E. Coccia ${ }^{13}$, A. Corvaglia ${ }^{14}$, D. De Gruttola ${ }^{15,16}$, S. De Pasquale ${ }^{15,16}$, F. Fabbri ${ }^{5}$, L. Galante ${ }^{17,18}$, M. Garbini ${ }^{10,12}$, G. Gemme ${ }^{6}$, I. Gnesi ${ }^{12,19}$, S. Grazzi ${ }^{6,20}$, D. Hatzifotiadou ${ }^{10,21}$, P. La Rocca ${ }^{22,23}$, Z. $\mathrm{Liu}^{24}$, G. Mandaglio ${ }^{20,23}$, G. Maron ${ }^{25}$, M. N. Mazziotta ${ }^{2}$, A. Mulliri ${ }^{8,9}$, R. Nania ${ }^{10}$, F. Noferini ${ }^{10}$, F. Nozzoli ${ }^{26}$, F. Palmonari ${ }^{10,11}$, M. Panareo ${ }^{14,27}$, M. P. Panetta ${ }^{12,14}$, R. Paoletti ${ }^{3,28}$, C. Pellegrino ${ }^{25}$, O. Pinazza ${ }^{10}$, C. Pinto ${ }^{22,23}$, S. Pisano ${ }^{5,12}$, F. Riggi ${ }^{22,23}$, G. C. Righini ${ }^{29}$, C. Ripoli ${ }^{15,16}$, M. Rizzi ${ }^{2}$, G. Sartorelli ${ }^{10,11}$, E. Scapparone ${ }^{10}$, M. Schioppa ${ }^{19,30}$, A. Scribano ${ }^{28}$, M. Selvi ${ }^{10}$, G. Serri ${ }^{8,9}$,

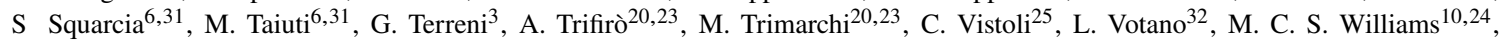
A. Zichichi ${ }^{10,11,21}$ and R. Zuyeuski ${ }^{24}$

${ }^{1}$ Dipartimento Interateneo di Fisica, Università di Bari, Bari, Italy. ${ }^{2}$ INFN Sezione di Bari, Bari, Italy. ${ }^{3}$ INFN Sezione di Pisa, Pisa, Italy. ${ }^{4}$ Dipartimento di Fisica, Università di Pisa, Pisa, Italy. ${ }^{5}$ INFN Laboratori Nazionali di Frascati, Frascati (Rome), Italy. ${ }^{6}$ INFN Sezione di Genova, Genova, Italy. ${ }^{7}$ Thomas Jefferson National Accelerator Facility, Newport News, VA, USA. ${ }^{8}$ Dipartimento di Fisica, Università di Cagliari, Cagliari, Italy. ${ }^{9}$ INFN Sezione di Cagliari, Cagliari, Italy. ${ }^{10}$ INFN Sezione di Bologna, Bologna, Italy. ${ }^{11}$ Dipartimento di Fisica ed Astronomia, Università di Bologna, Bologna, Italy. ${ }^{12}$ Museo Storico della Fisica e Centro Studi e Ricerche "E. Fermi", Rome, Italy. ${ }^{13}$ Gran Sasso Science Institute, LAquila, Italy. ${ }^{14}$ INFN Sezione di Lecce, Lecce, Italy. ${ }^{15}$ Dipartimento di Fisica, Università di Salerno, Salerno, Italy. ${ }^{16}$ INFN Gruppo Collegato di Salerno, Salerno, Italy. ${ }^{17}$ Dipartimento di Scienze Applicate e Tecnologia, Politecnico di Torino, Torino, Italy. ${ }^{18}$ INFN Sezione di Torino, Torino, Italy. ${ }^{19}$ INFN Gruppo Collegato di Cosenza, Cosenza, Italy. ${ }^{20}$ Dipartimento di Scienze Matematiche e Informatiche, Scienze Fisiche e Scienze della Terra, Università di Messina, Messina, Italy. ${ }^{21}$ CERN, Geneva, Switzerland. ${ }^{22}$ Dipartimento di Fisica e Astronomia, Università di Catania, Catania, Italy. ${ }^{23}$ INFN Sezione di Catania, Catania, Italy. ${ }^{24}$ ICSC World laboratory, Geneva, Switzerland. ${ }^{25}$ INFN-CNAF, Bologna, Italy. ${ }^{26}$ INFN Trento Institute for Fundamental Physics and Applications, Trento, Italy. ${ }^{27}$ Dipartimento di Matematica e Fisica, Università del Salento, Lecce, Italy. ${ }^{28}$ Dipartimento di Scienze Fisiche, della Terra e dell'Ambiente, Università di Siena, Siena, Italy. ${ }^{29}$ CNR Istituto di Fisica Applicata "Nello Carrara", Sesto Fiorentino (Florence), Italy. ${ }^{30}$ Dipartimento di Fisica, Università della Calabria, Rende (Cosenza), Italy. ${ }^{31}$ Dipartimento di Fisica, Università di Genova, Genova, Italy. ${ }^{32}$ INFN, Laboratori Nazionali del Gran Sasso, Assergi (L'Aquila), Italy. 